\title{
A Decentralized Damage Detection System for Wireless Sensor and Actuator Networks
}

\author{
Mili Verma \\ $M$. Tech (ECE), ABES Engineering \\ College, Ghaziabad, AKTU Lucknow, India
}

\author{
Himani Garg, PhD \\ Professor, ABES Engineering College, Ghaziabad, \\ AKTU Lucknow, India
}

\begin{abstract}
The miraculous capabilities of observing the physical world then processing the data, making decisions and performing appropriate actions of wireless sensor and actuator networks (WSAN) enable these networks to provide the several service oriented applications, such as environmental monitoring, industrial applications, security and surveillance, entertainment, smart building and healthcare(health monitoring and medical diagnostics). In such applications, our monitoring area empowered with wireless smart devices, are able to self-monitor and autonomously respond to situations using computational intelligence. This research focuses on developing a module to be setup in the hospitals to monitor the damage detection, which results delay on doctor and patient performances and also monitor WSAN sensor performances. This research work presents a decentralized algorithm for detecting damage by using a WSAN. Our algorithm makes use of cooperative information fusion for calculating a damage coefficient. Before the algorithm starts the network must be already deployed on the structure to be monitored. We found that our collaborative and information fusion-based approach ensures the accuracy of our algorithm . This is tolerable for maximum 100 nodes or less in the WSAN, operating system and protocols can last as long as 468 days.
\end{abstract}

\section{Keywords}

Wireless Sensor and Actuator, Decentralize Algorithm, Information Fusion, Hospital Monitoring.

\section{INTRODUCTION}

Advanced sensing systems play a major role as enabling technologies to build smart cities [2]. In smart cities, infrastructures, such as smart hospital and buildings, are equipped with smart sensing and actuator devices interconnected via wireless links composing a wireless sensor and actuator network (WSAN) [4]. The WSAN nodes are able to measure a variety of environmental parameters, process the sensing data locally, work in a collaborative way, make decisions on the occurrence of relevant events, and react to such events performing local control actions or sending warnings to remote operators. Applications running on top of WSAN are able to provide a wide variety of services to the citizens. An important application domain in smart cities is the smart hospital [3]. A smart hospital can be defined as a structure in which technologies and processes are used to increase enhanced patient care including remote medical care, enable efficient patient and medical information flow and boost diagnostic, surgical and organization intelligence capabilities while ensuring patient safety. The WSAN is an emerging technology, dealing with the development and implementation of continuous and reliable monitoring systems. The sensing devices collect data from the monitored environment, deliver them as digital data. Therefore, by processing this data, the co-operative information fusion algorithm techniques allow the detection, localization and extent determination of damage in WSAN nodes. Most of the wireless sensor network monitoring algorithms found in the literature employ centralized architectures with sensing nodes transmitting messages to a centralized entity wherein the damage detection processes effectively happen [5]. The major drawbacks of a centralized architecture is the, 1.Additional delay on top of control response time, because of the aggregated communication delay between sensor and actuator nodes. 2 .It minimizes WSAN lifetime. A Decentralize architecture is a feasible approach to overcome this architectural restriction to perform damage detection processes inside the WSAN nodes, which maximizes the WSAN lifetime and also improves wireless sensor network performance.

\subsection{Centralized and Decentralized Network System}

Table 1. Difference of Centralized and Decentralized System

\begin{tabular}{|l|l|}
\hline $\begin{array}{l}\text { Centralized network } \\
\text { system }\end{array}$ & Decentralized network system \\
\hline $\begin{array}{l}\text { 1. BS(basestation)collects } \\
\text { information from all the } \\
\text { sensor nodes. }\end{array}$ & $\begin{array}{l}\text { 1. Each sensor makes routing } \\
\text { decision based on their local } \\
\text { information. } \\
\text { 2. BS establishes routes. } \\
\text { 2..Route } \\
\text { establishment/management can } \\
\text { be much cheaper. }\end{array}$ \\
\begin{tabular}{l} 
4. Costly for installation. \\
\hline
\end{tabular} & \\
\hline
\end{tabular}

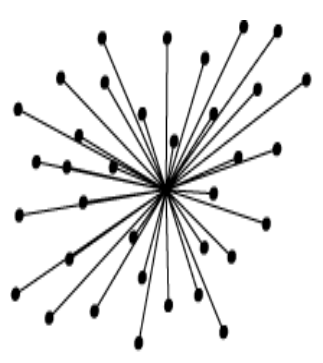

centralised

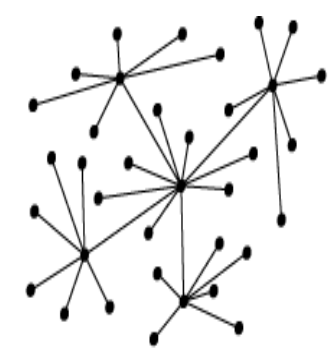

decentralised
Fig 1: Centralised \& Decentralised System

\subsection{WSAN (Wireless Sensor and Actuator Network) System}

Sensor is a transducer which converts physical phenomenon like heat, light, motion, vibration and sound into electrical signal. Sensor node is a basic unit in a sensor network which contains on board processors, transceivers, memory and power 
supply. Sensor network consist a large no. of sensor nodes. Nodes deployed either inside or very close to the sensed phenomenon.

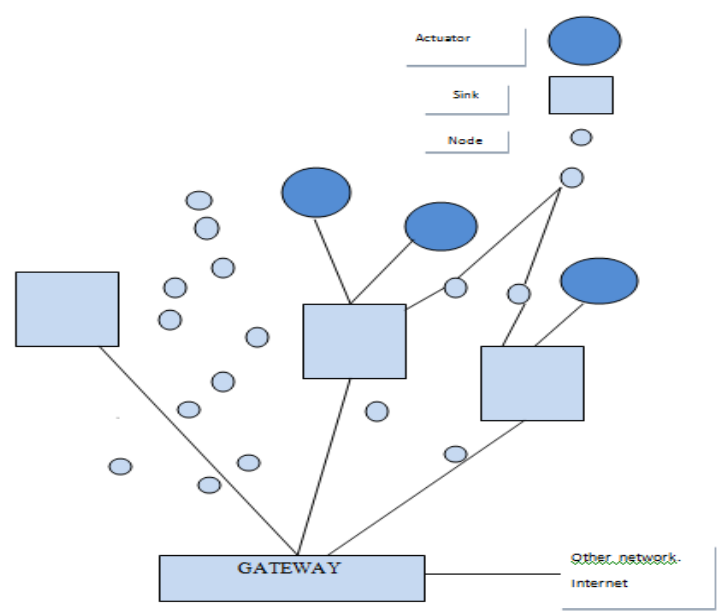

Fig 2: WSAN System

Wireless sensor observing the physical world(Sensors), processing the data, making decisions and performing appropriate actions(Actuators).

\section{RELATED WORK}

\subsection{Component Required}

\subsubsection{VM ware work station:}

Which was designed to run multiple operating systems at a same time on a desktop pc or system VM ware software runs on,

1. Microsoft windows

2. Linux

3. Mac Os

Advantages

1. Reduce physical infrastructure cost.

2. Reduce datacenter operating cost.

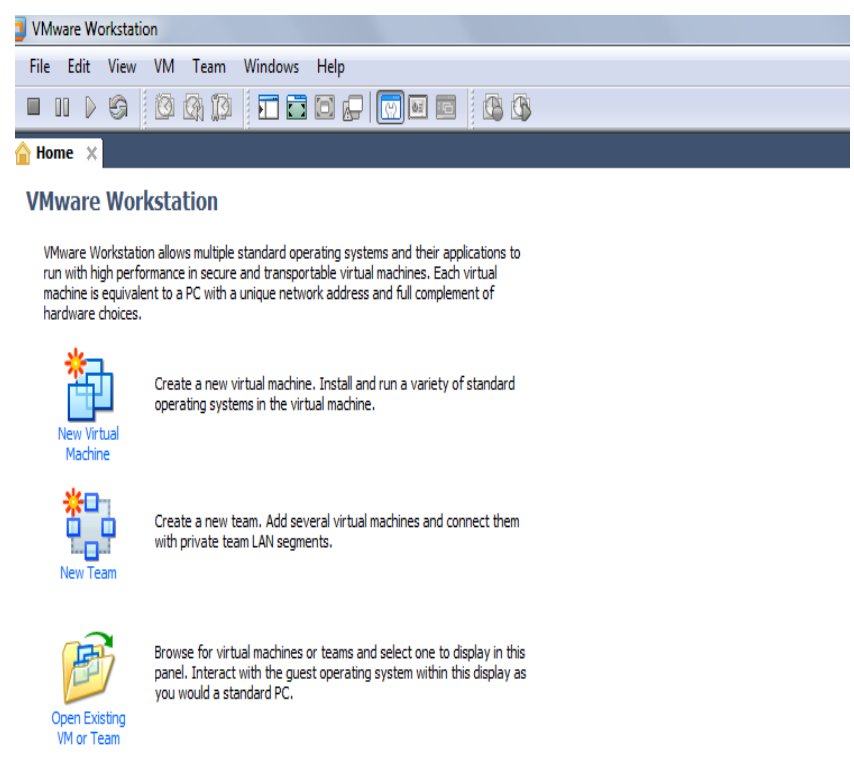

2.1.2 .Sensor Networks Operating System(Centos)

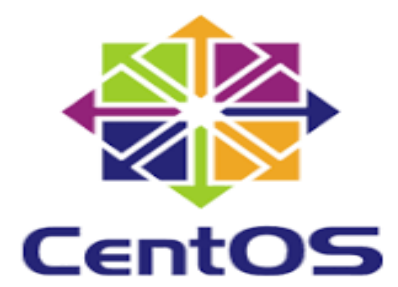

Table 2. Centos Description

\begin{tabular}{|l|l|}
\hline Centos & Description \\
\hline OS family & LINUX \\
\hline Source model & Open source \\
\hline Developer & $\begin{array}{l}\text { Affiliated with Red } \\
\text { Hat(American MNC) }\end{array}$ \\
\hline Kernal type & Monolithic(linux kernal) \\
\hline license & $\begin{array}{l}\text { Free software(GPL and other } \\
\text { license) }\end{array}$ \\
\hline
\end{tabular}

\subsubsection{Sensor Networks Simulator(NS2)}

System Simulator is fundamentally in view of two dialects. They are $\mathrm{C}++$ and $\mathrm{OTcl}$. OTcl is the question situated variant of Tool Command dialect. The system test system is a bank of various system and convention objects. $\mathrm{C}++$ helps in the accompanying way. It builds the productivity of recreation, used to give points of interest of the conventions and their operation.It is utilized to diminish parcel and occasion handling time. OTcl helps in the accompanying way.With the assistance of OTcl we can portray diverse system topologies, It encourages us to determine the conventions and their applications,It permits quick improvement, Tcl is good with numerous stages and it is adaptable for joining $\mathrm{Tcl}$ is anything but difficult to utilize and it is accessible in free.

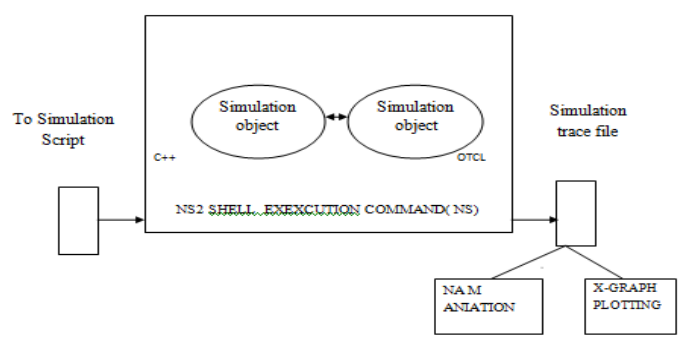

Fig 4: Basic architecture of NS

Fig 3: VMware workstation 
Table 3.NS2 Description

\begin{tabular}{|c|c|c|}
\hline Installation & Protocol & $\begin{array}{l}\text { Routing } \\
\text { agent }\end{array}$ \\
\hline $\begin{array}{l}\text { a)Ns2 is free simulation } \\
\text { tool, codes distributed in } \\
\text { two forms: }\end{array}$ & $\begin{array}{l}\text { The four ad-hoc } \\
\text { routing } \\
\text { protocols that } \\
\text { are currently } \\
\text { supported are : } \\
\text { 1.Destination } \\
\text { Sequence } \\
\text { Distance Vector } \\
\text { (DSDV), }\end{array}$ & $\begin{array}{l}\text { TCP-Transfer } \\
\text { control } \\
\text { protocol(used } \\
\text { for control } \\
\text { information) }\end{array}$ \\
\hline 1.The all-in-one suit & $\begin{array}{l}\text { 2.Dynamic } \\
\text { Source Routing } \\
\text { (DSR), }\end{array}$ & $\begin{array}{l}\text { FTP-File } \\
\text { transfer } \\
\text { protocol(used } \\
\text { for data } \\
\text { transfer) }\end{array}$ \\
\hline 2.The component wise & $\begin{array}{l}\text { 3.Temporally } \\
\text { ordered Routing } \\
\text { Algorithm } \\
\text { (TORA) }\end{array}$ & $\begin{array}{l}\text { UDP-User } \\
\text { datagram } \\
\text { protocol(used } \\
\text { to transfer } \\
\text { message from } \\
\text { one host to } \\
\text { another } \\
\text { without any } \\
\text { channel } \\
\text { requirement) }\end{array}$ \\
\hline $\begin{array}{l}\text { b)In this project all-in-one } \\
\text { suit is used which consist }\end{array}$ & $\begin{array}{l}\text { 4.Adhoc } \quad \text { On- } \\
\text { demand } \\
\text { Distance } \\
\text { (AODV) }\end{array}$ & $\begin{array}{l}\text { CBR- } \\
\text { Constant bit } \\
\text { rate(for } \\
\text { maximum } \\
\text { channel } \\
\text { capacity } \\
\text { used) }\end{array}$ \\
\hline $\begin{array}{l}\cdot \text { NS release } 2.30, \\
\cdot \text { Tcl/Tk release } 8.4 .13, \\
\text { - OTcl release } 1.12 \text {, and } \\
\cdot \text { TclCL release } \\
\text {-NAM(animation tool) } \\
\text {-Zlibversion1.2.3(required } \\
\text { lib for nam) } \\
\text {-Xgraph } \\
\text { plotter) }\end{array}$ & $\begin{array}{l}\text { 5Create protocol } \\
: \\
\text { lbegin }\{\text { program }\} \\
\text { \$opt(rp) } \\
\text { lend }\{\text { program }\}\end{array}$ & \\
\hline
\end{tabular}

\section{DESCRIPTION OF THE PROPOSED ALGORITHM}

A key challenge is how to maximize WSAN lifetime while maintaining the functionality of damage detection within the network. how to develop algorithms capable of supporting accurate damage detection, in terms of the rate of success in the detection of damage in monitoring area. Existing system (Damage detection algorithm ) Based only in the variation of modal frequency, it is less accurate because of single level collaboration procedure. Once in the Cooperative information fusion algorithm approach for damage detection, that are fully decentralize we are highlighting their advantages and drawbacks in relation to our work. The act of assessing information from two different sources simultaneously to make their own decision is called information fusion algorithm. In this damage co-efficient calculated from both amplitude and frequency shifts, decision inferred locally by each individual SEN node can also be exchanged among other WSAN nodes.Through specific collaboration procedures, the nodes can communicate their local decisions among themselves, evaluating their neighbor decisions and reaching a consensus, which may be more accurate than the single local decision of a node. A key characteristic of our algorithm is that it is based on the concept of cooperative information fusion. Moreover, a new damage coefficient, called cooperative damage coefficient (CDC), is proposed as a representation of local decisions made by each WSAN node. The CDC describes the damage using only a few bits in terms of both frequency and amplitude shifts simultaneously. which helps to achieve faster execution and enegy savings and also provide higher accuracy.

\section{MODULES IN IMPLEMENTATION}

\subsection{Network Deployment}

The procedures of our algorithm are performed by the set of WSAN nodes (excluding sink nodes) deployed over a monitoring area of a structure. We consider that each node may be equipped with both sensing and actuating devices. We consider two roles for the nodes:

$$
\begin{aligned}
& \text { 1. sensor (SENs) } \\
& \text { 2. actuator (ATNs). }
\end{aligned}
$$

\subsection{Setup Phase}

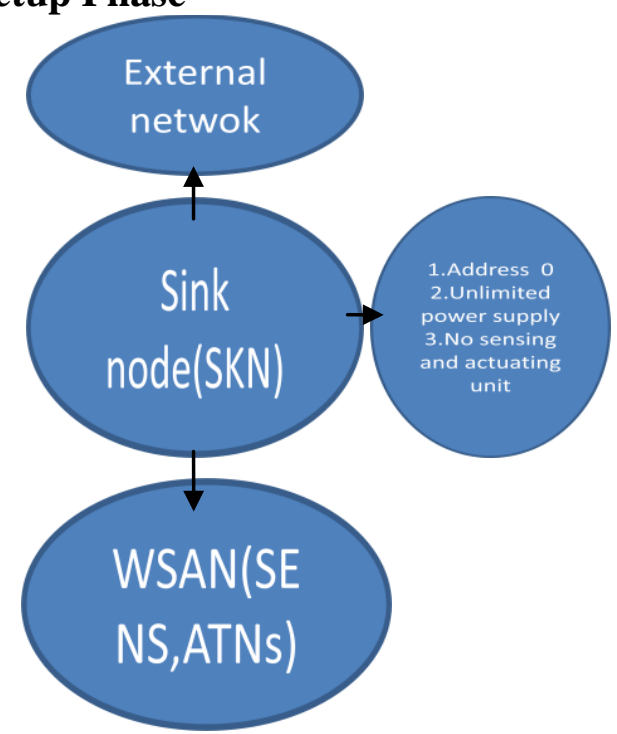

Fig 5:Setup phase

Such roles are set during the algorithm setup phase, when the respective logical capabilities of nodes are defined as active or non-active. A same node may have only the SEN role set, or only the ATN role or even both SEN and ATN roles simultaneously. A SEN is considered the basic "sensing, processing and decision unit" in the WSAN. Such nodes are equipped with at least one physical sensing device, and have their logical sensing capability active. Similarly, each ATN is 
considered the basic "actuation unit" in the WSAN. Such nodes are equipped with at least one physical actuation device. Both SEN and ATN nodes have unique non-zero network addresses. We identify the single sink node (SKN) in the network by the address 0 , being also a role set during the setup phase. The SKN serves as a gateway between the WSAN and external networks. It has no sensing units and unlimited power supply. Therefore, the SKN is an intermediate between the human operators and the WSAN nodes, disseminating commands in the WSAN and collecting reports from actions performed on the structure. Finally, before the algorithm starts, the network must be already deployed on the structure to be monitored.

1.be enough to ensure a good resolution in the power spectrum that will be returned, which implies in better precision in the modal frequencies and amplitude determination;

2. be a power of 2 , since this is a requirement for the entry of data in the FFT algorithm;

3. not exceed the sensors storage capacity (Flash memory).

Sample rate must be:

1. greater than the value of the first modal frequencies of interest so that these are shown in the power spectrum.

2. high enough to ensure accuracy.

3. twice the highest modal frequency of interest, to meet the Nyquist criterion. neighbouring node NeNodeID is a data structure, defined for each node, whose elements store the unique network addresses (NodeID) of all the neighbor nodes. The first position of the NeNodeID data structure stores the amount of neighbors of each node.

\subsection{Steps To Define Wireless Sensor Network Parameter}

Table 4. WSAN Parameter

\begin{tabular}{|l|l|l|}
\hline Setval (chan) & $\begin{array}{l}\text { Channel/wireless } \\
\text { channel }\end{array}$ & \#channel type \\
\hline Setval (prop) & $\begin{array}{l}\text { Propagation two } \\
\text { way ground. }\end{array}$ & \#radio propagation \\
\hline Setval (ant) & $\begin{array}{l}\text { Antenna/Omni } \\
\text { directional antenna }\end{array}$ & :\#antenna type \\
\hline Setval (LL) & LL & $: \#$ Link layer type \\
\hline Setval (ifq) & Queue/Droptail & :\#Interface type \\
\hline Setval (nn) & 80 & :\#Number of nodes \\
\hline Setval (ifqlen) & 4000 & in a queue type \\
\hline
\end{tabular}

\begin{tabular}{|c|c|c|}
\hline Setval (mac) & $\begin{array}{l}\text { Mac/802.11 and } \\
\text { TDMA Mac }\end{array}$ & :\#Mac type \\
\hline Set val (rp) & $\begin{array}{l}\text { DSDV,AODV,DS } \\
\text { R,TORA }\end{array}$ & $\begin{array}{ll}\text { :\#Ad-hoc routing } \\
\text { protocol }\end{array}$ \\
\hline
\end{tabular}

\subsection{Topology (Cluster based topology)}

In our research WSAN hierarchically divided into clusters, reducing the amount of transmissions in the network, since it is not necessary that all sensors forward their data to the sink. At the same time, since the amount of data sent to the sink is smaller and already condensed, there is a reduction in the time of damage identification. A system topology is the course of action of a system, including its hubs and associating lines. There are two different ways of characterizing system geometry: the physical topology and the coherent (or flag) topology.

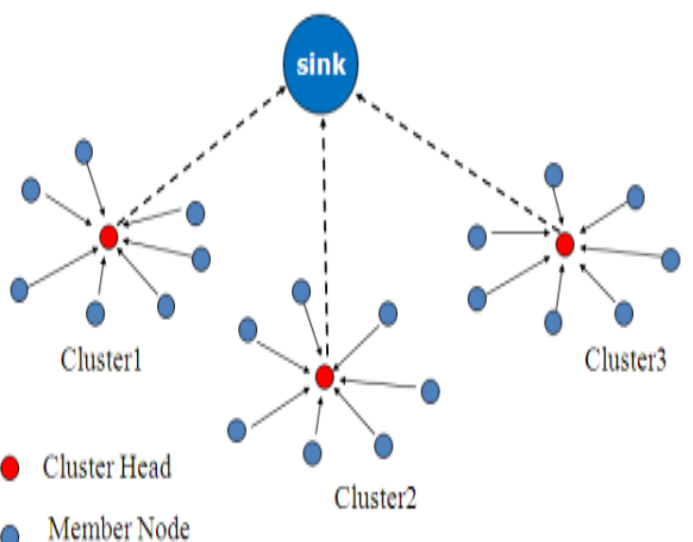

Fig 6: Cluster based topology 


\subsection{Monitoring Cycle Phase}

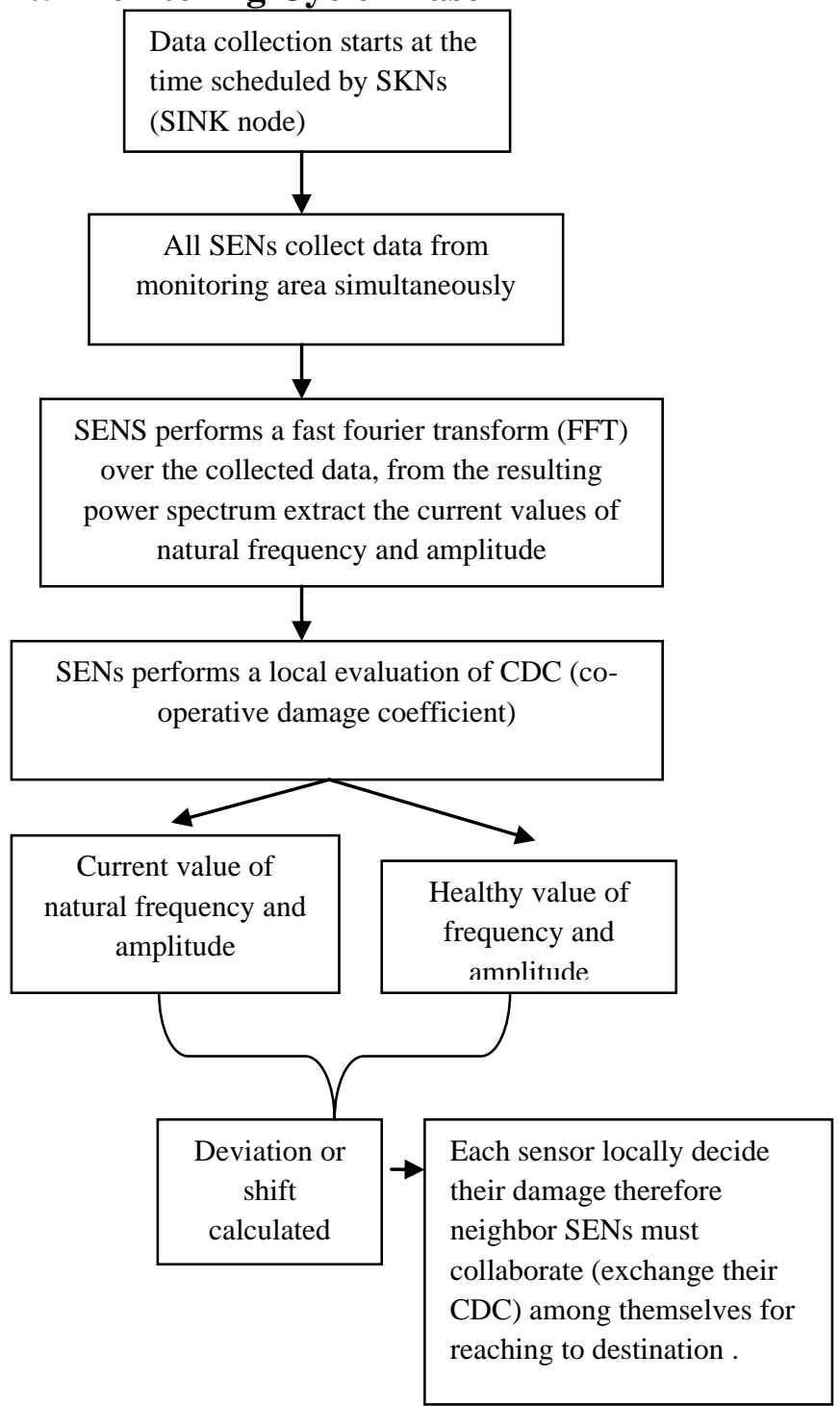

\section{RESULT AND ANALYSIS}

A decentralize damage detection system for wireless sensor and actuator network .Fig 7 shows Network animation trace, in this all the nodes are movable and each node performing different function. There is one source and one destination node .NODES will move from source to destination .Fig 8 represents the graph between WSAN nodes (X-axis) and communication with doctor(Y-axis).Fig 9 shows the graph between no. of WSAN nodes(X-axis )and no. of patient(Yaxis),which shows wireless sensor and actuator network performance.

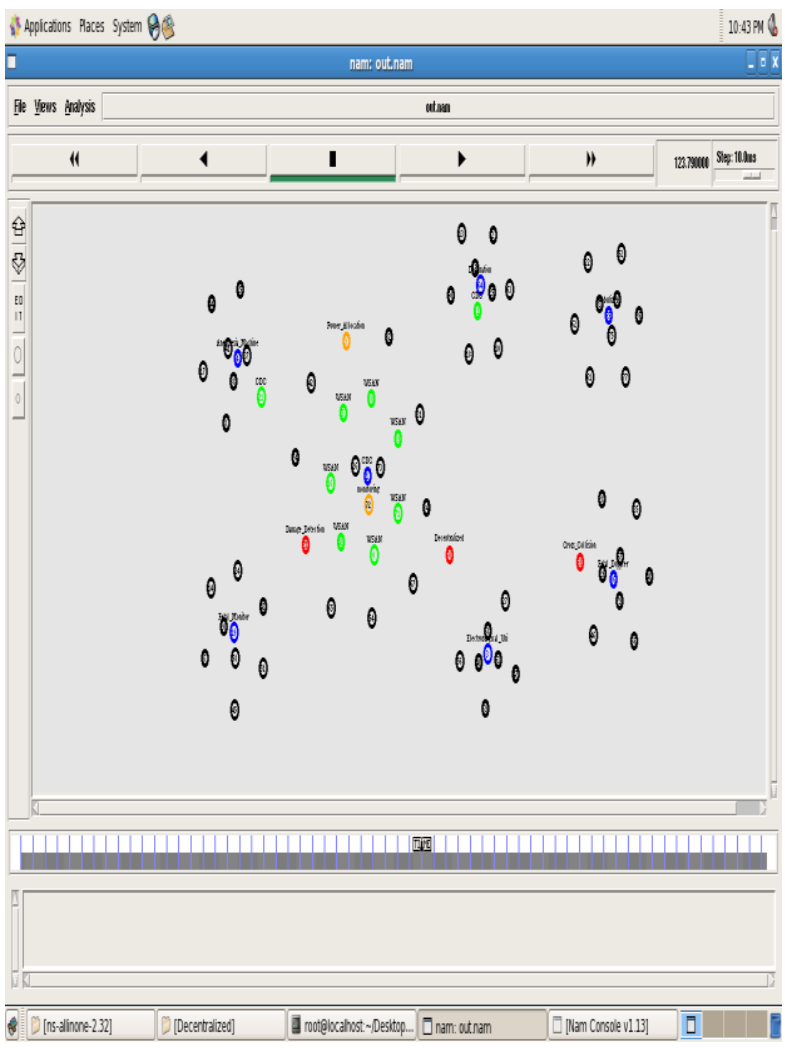

Fig7: Monitoring Area (Output NAM trace)

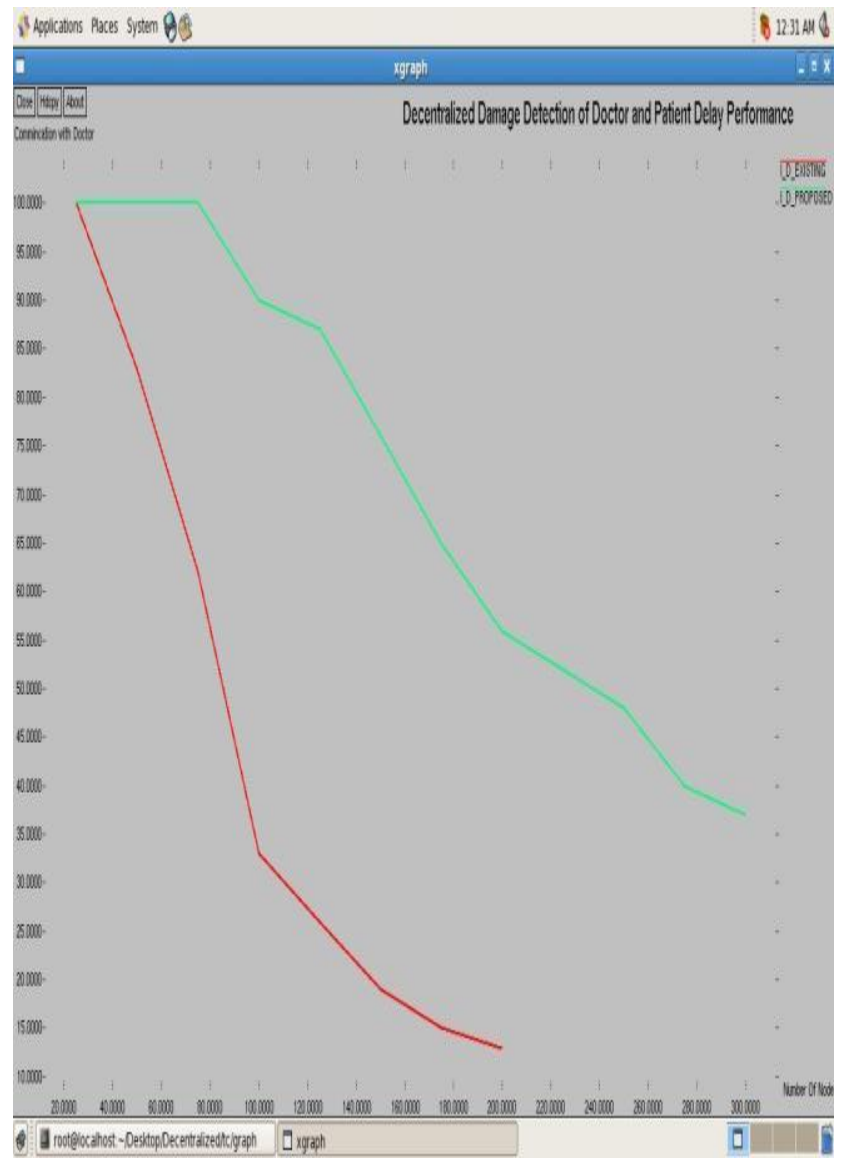

Fig 8: Decentralize Damage Detection of Doctor Patient Delay Performance 


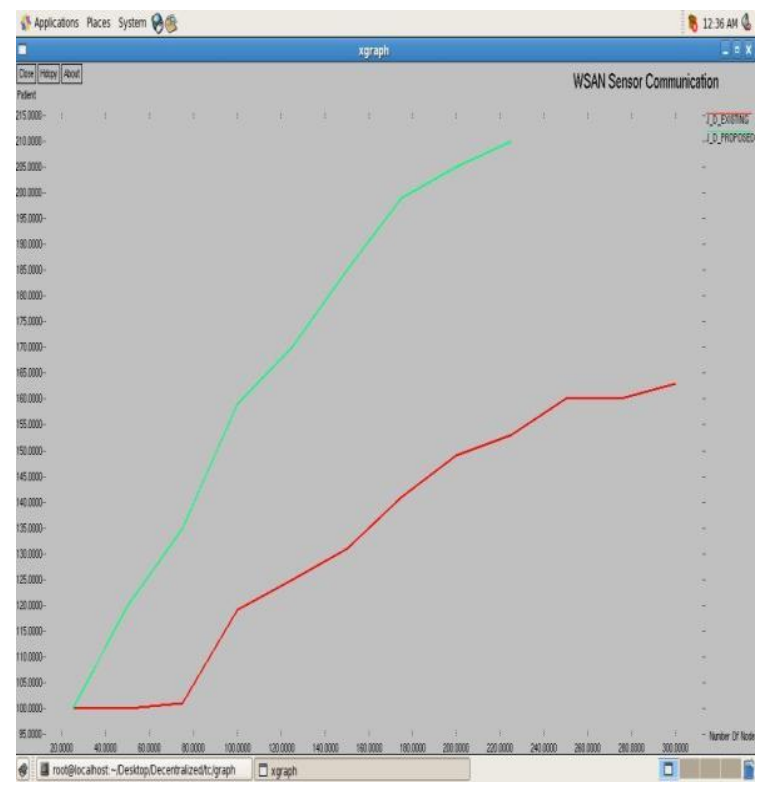

Fig 9: Graph for WSAN Sensor communication

\section{CONCLUSION AND FUTURE SCOPE}

This research paper exhibited a decentralized process for identifying damage in the monitoring area utilizing WSAN nodes. Our work has helped to reduce data transmission. Which improves the overall performance of the wireless sensor also helps maximize the lifetime of WSAN. In future work, we mean to investigate the fusion of data alongside information from various kinds of sources. Future course is to consider the presence of an ideal position, where the lessening of information and data ought to be connected in our assessment. At long last, we might want to watch that our reenactment ponders not just show the great execution capability of the proposed approach, however the reproduction models themselves give an intense structure to model ingand dissecting the conduct (execution) of the proposed blame tolerant systems, in this manner furthermore delineating the energy of recreation considers. For intermittently damage tolerant /benefit survivability systems can not be assessed since they might not have shut shape arrangements as well as be tractable. Nonetheless, it turns out to be imperative to break down the execution of a proposed self-mending system keeping in mind the end goal to see its helpfulness. It is in such situations that we see the significance of good demonstrating and reproduction thinks about, and consequently, notwithstanding proposing a self-recuperating instrument, the work in this paper is utilized to outline the significance of recreation based investigations for dissecting and assessing complex frameworks and plans.

\section{ACKNOWLEDGMENTS}

Primarily we would thank god for being able to complete this, then thanks to my thesis advisor Dr. Hiani Garg, who have contributed towards development of this paper.

\section{REFERENCES}

[1] Citation information: DOI10.1109/TC.2015.2479608, IEEE Transactions on Computers.

[2] R. Giffinger, C. Fertner, H. Kramar, R. Kalasek, "Smart Cities: Ranking of European Medium-Sized Cities," Centre of Regional Science, Vienna UT, Vienna, Austria, Tech. Rep., Oct. 2007.
[3] L. Schor, P. Sommer, R. Wattenhofer, "Towards a zeroconfiguration wireless sensor network architecture for smart buildings," In Proc. SenSys09, 2009, pp. 31-36.

[4] I. Akyildiz, I. Kasimoglu, "Wireless sensor and actor networks: Research challenges," Ad Hoc Networks, vol. 2, no. 4, pp. 351-367, Oct. 2004.

[5] G. Hackmann, F. Sun, N. Castaneda, C. Lu, S. Dyke, "A holistic approach to decentralized structural damage localization using wireless sensor networks," Computer Communications, vol. 36, no. 1, pp. 29-41, Dec. 2012.

[6] L.Gu and J.A. Stankovic, "t-kernel: Providing Reliable OS Support for Wireless Sensor Networks," Proc. ACM Conf. on Embedded Networked Sensor Systems (Sensys), 2006, pp. 1-14.

[7] P. Kamat et al., "Enhancing Source Location Privacy in Sensor Network Routing." Proc. Int'l Conf. Distributed Computing Systems, 2005, pp. 559-608

[8] R. Verdone et al., Wireless Sensor and Actuator Networks: Technologies, Analysis and Design, Academic Press, Jan. 2008.

[9] A. Srinivasan, J.Teitelbaum, J. Wu, "DRBTS: Distributed Reputation-Based Beacon Trust System," 2nd IEEE Int'l Symp. Dependable, Autonomic, and Secure Computing (DASC), 2006, pp. 277-283.

[10] N. Ramanathan et al., "Sympathy for the Sensor Network Debugger," Proc. the 3rd ACM Int'l Conf. Embedded Networked Sensor Systems (Sensys), 2005, pp. 255-267.

[11] H. Oliveira, A.Boukerche, E.Nakamura, A. Loureiro, "An efficient directed localization recursion protocol for wireless sensor networks," IEEE Trans. Computers, vol. 58, no. 5, pp. 677-691, Dez. 2008, doi: 10.1109/TC.2008.221.

[12] R. Kay and F. Mattern, "The Design Space of Wireless Sensor Networks" IEEE Wireless Communications Vol.11.No.6 (2004) pp 54-61.

[13] A. Tiwari, P. Ballal and F.L. Lewis, "Energy efficient wireless sensor network design and implementation for condition based maintenance" ACM Transactions on Sensor Networks (TOSN) Vol.3.No.1 (2007) pp 17.

[14] Aminian M, Naji HR (2013) A Hospital Healthcare Monitoring System Using Wireless Sensor Networks. J Health Med Inform 4:121. doi: 10.4172/21577420.1000121

[15] D.Malan, T.R.F.Fulford-Jones, M.Welsh,S.Moulton, CodeBlue: an ad-hoc sensor network infrastructure for emergency medical care, in: Proceedings of the Mobi-Sys 2004 Workshop on Applications of MobileEmbedded Systems (WAMES2004), Boston,MA, June,2004, pp.1214

[16] N. Wang, M. H. Wang and N. Q. Zhang, "Wireless sensors in agriculture and food industry: Recent development and future perspective" Computers and electronics in agriculture Vol.50.No.1 (2006) pp 114

[17] M.C. Domingo and R. Prior, "Energy analysis of routing protocols for underwater wireless sensor networks" Computer Communications. Vol.31.No.6 (2008) pp 
12271238.

[18] AJG Sanchez, F. GarciaSanchez, F. Losilla, P. Kulakowski, J. GarciaHaro, A. Rodríguez, JV LópezBao and F. Palomares Article:Wireless Sensor Network Deployment for Monitoring Wildlife Passages Sensors, Vol.10.No.8 (2010) pp 72367262.

[19] E. C.-H. Ngai, M. R. Lyu, and J. Liu, "A real-time communication framework for wireless sensor-actuator networks," in Proc. of the IEEE Aerospace Conference, Big Sky, Montana, U.S., Mar 2006.
[20] G.Hackmann, F. Sun, N. Castaneda, C. Lu, S. Dyke, "A holistic approach to decentralized structural damage localization using wireless sensor networks," Computer Communications, vol. 36, no. 1, pp. 29-41, Dec. 2012.

[21] R.Klempous, J. Nikodem, L. Radosz, N. Raus, "Byzantine Algorithms in Wireless Sensors Network," in Proc. ICIA, 2006, pp. 319-324.

[22] E. Nakamura, A. Loureiro, A. Frery, "Information fusion for wireless sensor networks: Methods, models, and classifications," ACM Comput. Surv., vol. 39, no. 3, pp. 9/1-9/55, Sep. 2007. 